\title{
Analysis of crystal growth kinetics in undercooled melts by infrared thermography
}

\author{
by A. Godin*, M. Duquesne*, E. Palomo del Barrio*, J. Morikawa**.
}

* Univ Bordeaux, I2M, CNRS, UMR 5295, F-33400 Talence, France. CNRS, I2M, UMR 5295, F-33400 Talence, France. Arts et Metiers ParisTech, I2M, UMR 5295, F-33400 Talence, France, alexandre.godin@ensam.eu

** Tokyo Institute of Technology, 2-12-1, S8-29, Okayama, Meguro-ku, Tokyo 152-8550 Japan.

\begin{abstract}
A new experimental approach based on infrared thermography is proposed in this paper for studying crystal growth kinetics in undercooled melts. The crystallization of a thin sample of an undercooled melt at constant bulk temperature is induced by a small crystal seed and the growth of the solid phase is observed using infrared camera. The recorded thermal images allow determining the position of crystallization front at any time, the velocity of advancement of the front at any point and time, and the temperature at the points of the interface at any time. Contrary to experimental techniques based on optical microscopy or video cameras, infrared thermography provides detailed analysis of the interface temperature which is essential when discussing the temperature dependence of experimentally determined growth rates. The appropriateness of infrared thermography for crystal growth kinetics analysis is illustrated through the experimental analysis of erythritol crystallization.
\end{abstract}

Key words: Crystal growth, crystal morphology, infrared thermography

\section{Introduction}

The formation of complex microstructures during solidification of metals and alloys has fascinated researchers in material sciences and related areas for hundreds of years. Recent advances in atomistic and phase-field modeling as well as new experiments have led to major progress in the field during the first years of this century [1]. However the basic problem of understanding and controlling microstructures and growth kinetics during solidification continues to present numerous scientific and technological challenges.

An important problem, which is arising in considering the solidification problems, concerns the kinetics of the advancement of the solid-liquid interface. The rate at which the crystal grows can be controlled by any of three factors: species diffusion in the melt (either long- or short-range), flow of latent heat away from the growing crystal surface or reactions at the crystal-melt interface. In most cases the experimental procedure for measuring growth rates is to produce an homogeneous melt, quench it to a glass, reheat the glass to the desired temperature in standard resistance furnaces, and measure the length of the crystals nucleated on the surface of the specimens as a function of time at constant temperature. In some cases, especially in systems which crystallize at low temperatures, the rates are measured using microscope heating-cooling stages by direct observation of the crystals as a function of time at constant temperature. A large range of growth rates has been experimentally measured by these techniques, from very low growth rates, such as those of certain proteins $\left(10^{-9} \mathrm{~m} \cdot \mathrm{s}^{-1}\right)$, to high growth rates for metals $\left(10^{-1} \mathrm{~m} . \mathrm{s}^{-1}\right)$.

Time-laps photography or cinematography methods using either optical microscopy or video cameras are the most widely used imaging techniques $[2,3]$. They allow analyzing both the crystal morphology and the crystal growth rate as a function of the undercooling. However, the only thermal information provided by these experimental approaches is the temperature in the bulk of the specimens (e.g. temperature of the growth cell, temperature of the support on which the specimen is placed), which is usually lower than that of the crystallization front. If the rate of production of latent heat is faster than the rate of removal, the latent heat release at the interface will cause the temperature to rise until the growth rate slows down to the point where the latent heat can be removed at the same rate it is produced. Thus, the growth rate is ultimately controlled by either species diffusion or interface reaction, but the local temperature at the interface is higher than the bulk temperature. Several authors have used this argument to explain the discrepancies observed between atomistic simulations and experimental values regarding the temperature dependency of the crystals growth rates $[4,5]$. In simulations the latent heat of fusion can be removed quickly from the system by an applied thermostat, whereas in experiments the heat must be removed by a thermal diffusion process. Therefore, a detailed analysis of the interface temperature is essential when discussing the temperature dependence of experimentally determined growth rates.

Experimental analysis of crystal growth in undercooled melts by using microscopic infrared thermography instead of optical microscopy is proposed in this paper. Infrared camera can provide thermal images of the specimen at high scan speed $(>1600 \mathrm{~Hz}$ ), thus allowing determining the temperature at any point of the crystal-melt interface at any time. The spatial resolution of infrared images is however limited by the wavelength of infrared radiation and it is hence lower than that of the images obtained by optical microscopy. In practice the highest resolution reachable with conventional lenses is about $20 \mu \mathrm{m}$. Optical microscopy will hence provide a better view of the crystal morphology than infrared thermography, whereas the latter one will be preferred for analyzing the temperature dependence of crystal growth rates.

*Corresponding Author: alexandre.godin@ensam.eu 
The paper is organized as follows. In section 2, the theory of interface-controlled crystal growth is recall. The experimental setup and the experiments proposed for crystal growth rates analysis are described in section 3 . Data processing is explained in section 4. The crystal growth of undercooled erythritol is experimentally studied in the last section.

\section{Recall on interface-controlled crystal growth}

If there is no phase separation upon crystallization (e.g. pure substances, eutectic mixtures), the composition of the parent phase and crystal are the same at all the times. Therefore, in experiments at constant bulk temperature growth is controlled by rearrangement processes only at the liquid-solid interface and is therefore interface-controlled. Such a rearrangement process may either involve a diffusive jump in the case of diffusion-limited kinetics or an atomic collision in the case of collision-limited kinetics. The rearrangement frequency is in both cases independent of the interface position so that the growth velocity is time-independent.

The description of kinetic effects during crystal growth in deeply undercooled melts can be described using the rate theory [6, 7]. It is assumed that the attachment of a single atom from the liquid to the crystallization front takes place via atomic diffusion processes over an activation threshold, which is built up by the energy potential of the surrounding atoms. Usually, it is assumed that the energy barrier corresponds to the activation energy for atomic diffusion. Calculating the rates with which the atoms undergo a transition from the liquid to the solid state, $r_{\mathrm{s}}$, and vice versa the backward reaction, $r_{l}$, by Arrhenius law, the crystal growth velocity can be written as:

$$
v=a_{o} f\left(r_{l}-r_{s}\right)=a_{o} f v^{\prime} \exp \left(-\frac{\Delta G_{a}}{R T}\right)\left[1-\exp \left(-\frac{\Delta G_{l s}}{R T}\right)\right]=v_{o}\left[1-\exp \left(-\frac{\Delta G_{l s}}{R T}\right)\right]
$$

$a_{o}$ is assumed to be the typical interatomic spacing, $f$ is the fraction of interface sites that are active growth sites and $v^{\prime}$ characterizes a typical atomic vibration or thermal frequency of single atoms in the liquid state. $\Delta G_{l s}=G_{l}-G_{s}$ is the local free energy or chemical potential difference between the two phases that is driving the transformation, whereas $\Delta G_{a}$ corresponds to the activation energy for atomic jumps. $R$ is the universal gas constant and $T$ the temperature. $v_{0}$ represents the speed limiting the attachment of atoms at the interface. At constant pressure:

$$
\Delta G_{a}=\Delta H_{a}-\Delta S_{a} T ; \quad \Delta G_{l s}=\Delta H_{l s}-\Delta S_{l s} T
$$

where $S$ and $H$ represent the entropy and the enthalpy respectively. Moreover, by assuming that the enthalpy and entropy differences are not sensitive functions of the temperature, $\Delta G_{/ s}$ may be approximated by

$$
\Delta G_{l s}=\Delta H_{l s} \Delta T / T_{m}
$$

where $\Delta H_{l s}$ is the latent heat of fusion, $\Delta T=T_{m}-T$ is the undercooling and $T_{m}$ is the liquidus temperature.

The early models for the growth rate of a crystal from a liquid are those of Wilson in 1900 and Frenkel in 1930, in which the factor $v_{0}$ in Eq. 1 is related to atomic diffusivity $D \sim \exp \left(-\Delta H_{a} / R T\right)$. The modern form of the Wilson-Frenkel model is [6]:

$$
v=\frac{6 a_{o} D(T)}{l^{2}} f \exp \left(-\frac{\Delta S_{a}}{R}\right)\left[1-\exp \left(-\frac{\Delta G_{l s}}{R T}\right)\right]
$$

where $/$ is the diffusion jump distance. The diffusion coefficient can be expressed via the Stokes-Einstein relation:

$$
D(T)=\frac{k_{B} T}{3 \pi \eta(T) a_{o}}
$$

$k_{B}$ is the Boltzmann constant and $\eta$ is the viscosity of the liquid.

Equations (4)-(5) are appropriate expressions for the crystallization rate of glass-forming materials, where the mobility of the atoms limits the growth rate (diffusion-limited kinetics). In these materials a rearrangement of the configuration and positions of the other atoms in the liquid is required in order an atom or molecule join the crystal. This rearrangement process is thermally activated with the same activation energy as liquid diffusion and viscosity. The crystal growth rate is zero at the liquidus, increases to a maximum, and then decreases as the activation energy term begins to dominate.

In materials for which no rearrangement of the liquid structure is required for any individual atom to join the crystal (e.g. pure metals), the crystallization is not thermally activated and equations (4)-(5) don't apply. Recent molecular dynamics simulation results $[6,7]$ show that crystal growth is limited by atomic collisions (collision-limited kinetics) and the following expression has been proposed for calculating the growth velocity: 


$$
v=\frac{a_{o} f}{l^{2}} \sqrt{\frac{3 k_{B} T}{m}} \exp \left(-\frac{\Delta S_{a}}{R}\right)\left[1-\exp \left(-\frac{\Delta G_{l s}}{R T}\right)\right]
$$

where $m$ is the atomic mass. In these materials, crystals can grow even at effectively zero values of diffusivity.

In both cases, diffusion-limited and collision-limited kinetics, the fraction $f$ of interface sites that are active growth sites is generally not known. $f$ is typically about 0.25 for a rough surface, but can be very small on a smooth surface. Moreover, this factor is strongly temperature dependent if the growth depends on the nucleation of a new layer. Therefore, equations (3) or (4) are often used in crystal growth experimental studies to investigate the temperature dependence of $f$.

\section{Description of the experimental setup and the experiments}

\subsection{Experimental setup}

A schematic drawing of the experimental set-up is shown in Figure 1. The main elements of the set-up are an infrared camera and a heating/cooling system for temperature control. The heating/cooling system is a layered structure including an insulation layer at the bottom, a flat heating element in the middle and a thin plate made of aluminium painted in black on the top. The aluminium plate acts as thermal diffuser and it is equipped with a thermocouple for temperature control. The infrared camera (FLIR SC7000, detection window: 1.5-5 $\mu \mathrm{m}$ ) is equipped with an optical microscope LO709T. In practice, the highest spatial resolution reachable is about $20 \mu \mathrm{m}$. Digital full frame rate of the camera ranges from $170 \mathrm{~Hz}$ to $380 \mathrm{~Hz}$, and up to $11 \mathrm{kHz}-32 \mathrm{kHz}$ with windowing. The set-up is mounted on a vibration free solid support. Allowed working temperatures ranges from ambient temperature to $200^{\circ} \mathrm{C}$.

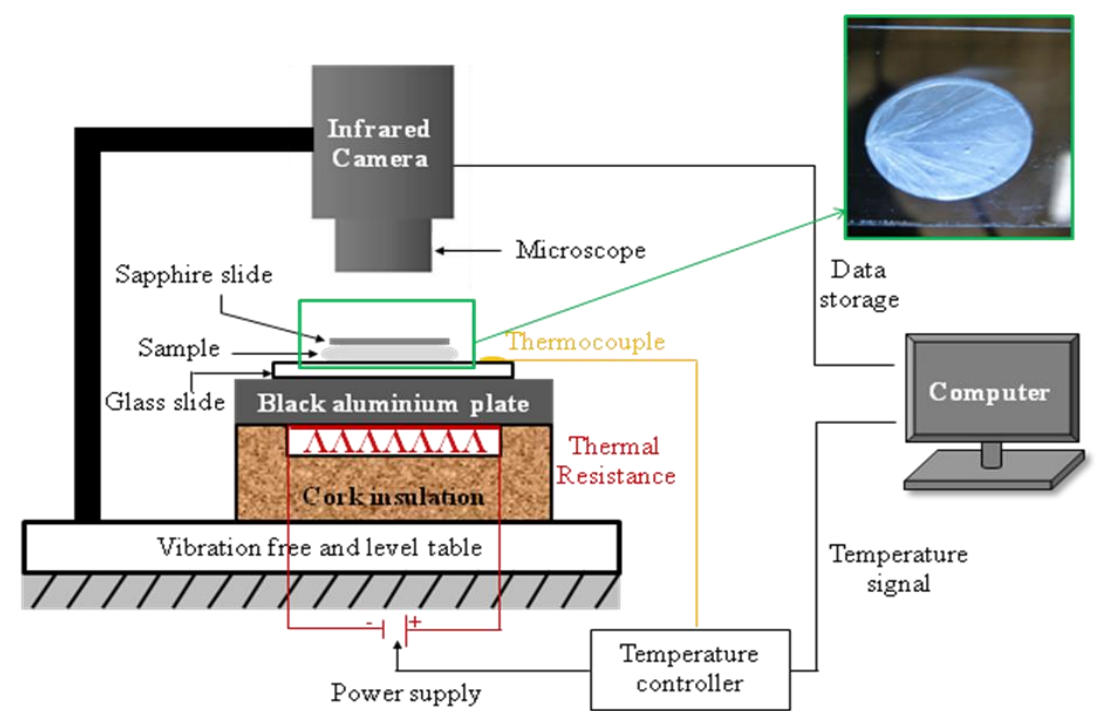

Fig. 1. Sketch of the experimental device and picture of a sample (green box)

\subsection{The sample and the experiments}

The sample is a layer of the test product which is in the liquid state at temperature below the melting point (undercooled melt). The undercooled melt is placed between a small glass slide (bottom side) and a sapphire slide (top side) which is transparent to infrared wavelengths. This sandwich is prepared as follows: 1 ) the product is first molten in a vessel; 2) the molten product is then poured on the glass slide; 3 ) at last, the sapphire slide is put on the top of the product and the distance between the slides is adjusted. The sample can be $300 \mu \mathrm{m}$ to $1 \mathrm{~mm}$ of thickness.

The tests are carried at constant temperature. The heating/cooling system is used to reach the selected working temperature $\left(T_{0}\right)$ on the aluminium plate and to keep it constant thereafter. The sample, sandwiched as described above, is then placed on the aluminium plate and we wait until thermal equilibrium is reached. Afterwards, the crystallization is initiated with a small seed of the studied product. For this, the seed is placed at the edge of the sapphire slide in contact with the undercooled melt. The thermal behavior of the sample during crystallization is observed by the infrared camera. Simultaneously, the temperature of the glass slide is measured by a thermocouple.

We remind that the infrared camera doesn't provide direct measurements of temperature, but electrical signals $(D L=$ Digital Level) which are proportional to the photonic flux received by the infrared sensors. A calibration is hence necessary to transform $D L$ values into temperature data. Therefore, once the sample is placed on the experimental setup and reach thermal equilibrium, a series of 1000 images is recorded using the infrared camera and the temperature of the sample is measured by the thermocouple which is placed on the surface of the glass slide. This procedure is repeated for different values of the working/sample temperature. The calibration curve is then obtained by representing the spacetime average of DL-values in a series of images against the corresponding sample temperature (see e.g. Fig. 4).

The calibration is carried out with the product in liquid state (undercooled melt) and, consequently, it does not applies to the solid phase. Figure 2 (left side) shows the infrared image of two samples of the same product, one is in 
liquid state (orange area, top side) and the other is solid (blue/green area, bottom side). Although both are in thermal equilibrium at the same temperature, the photonic flux received by the infrared camera from the sample in liquid state is higher than the one received from the crystallized sample due to the differences in emissivity. It can also be noticed that the photonic flux is quite uniform when the sample is liquid whereas it is not in the solid phase. Moreover, the morphology and the orientation of the crystals in the solid phase can be very sensitive to the experimental conditions (e.g. size and form of the seed) and be different from one experiment to another as shown in Fig. 2. Specific calibration procedures for the solid phase must hence be defined.
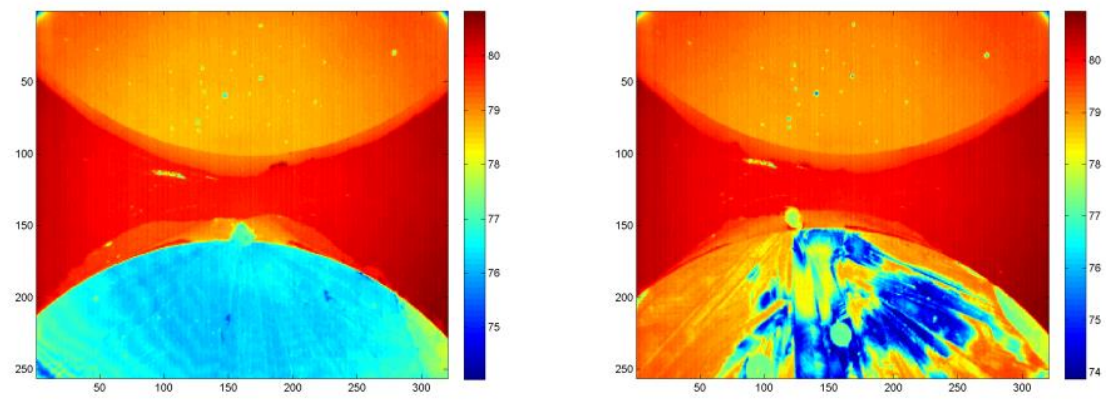

Fig. 2. Comparison of the images recorded by the infrared camera at constant temperature $\left(80^{\circ} \mathrm{C}\right)$ for the liquid (semi circle at the top side) and solid (semi circle of the bottom).

\section{Data processing to determine the crystal growth velocity from the infrared images}

\subsection{Raw data}

Let us consider an experiment carried out at working temperature $T_{0}$. This temperature (far-field temperature hereafter) is measured by the thermocouple placed on the surface of the glass slide and remains constant during the experiment. Data recorded by the infrared camera are DL maps (300 x 250 pixels) with lateral resolution around $20 \mu \mathrm{m}$. Images are recorded at $27 \mathrm{~Hz}$. Figure 3 provides an example of DL maps obtained at different times during the experiment. White color corresponds to the solid-liquid interface, the liquid phase is in black and the solid phase is grayscale.
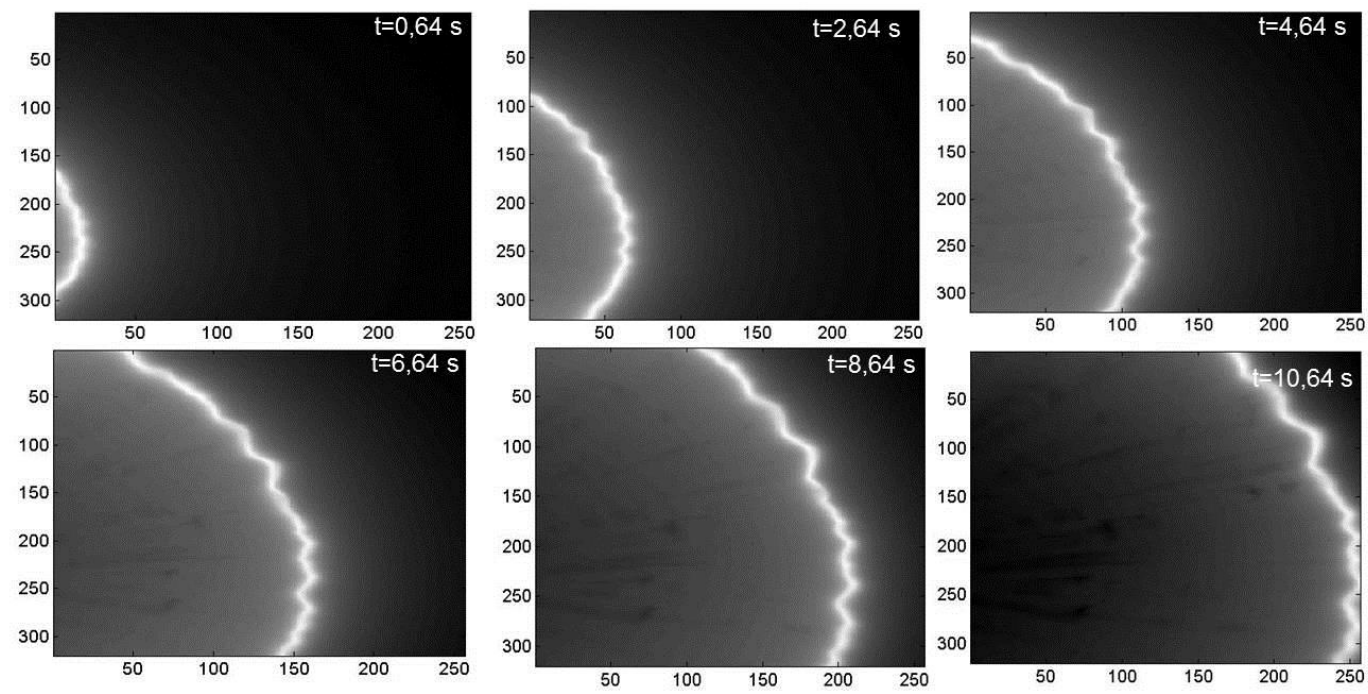

Fig.3. Snapshots of the infrared images recorded over time.

\subsection{Interface tracking and interface temperature}

DL data are transformed into temperature values using the calibration curve determined before. At any time, the maximum values of temperature have to be at the solid-liquid interface where crystallization and, consequently, latent heat release, is taking place. At each sampling time, the interface position $s(x, y, t)$ is hence determined by searching the maximum value of temperature in each row of the corresponding thermal image. Figure 4 shows the calibration curve for the data in Fig.3, the thermal image obtained by applying this transformation (DL into ${ }^{\circ} \mathrm{C}$ ) to the infrared image recorded at $8.64 \mathrm{~s}$, and the corresponding solid-liquid interface determined as described above. 

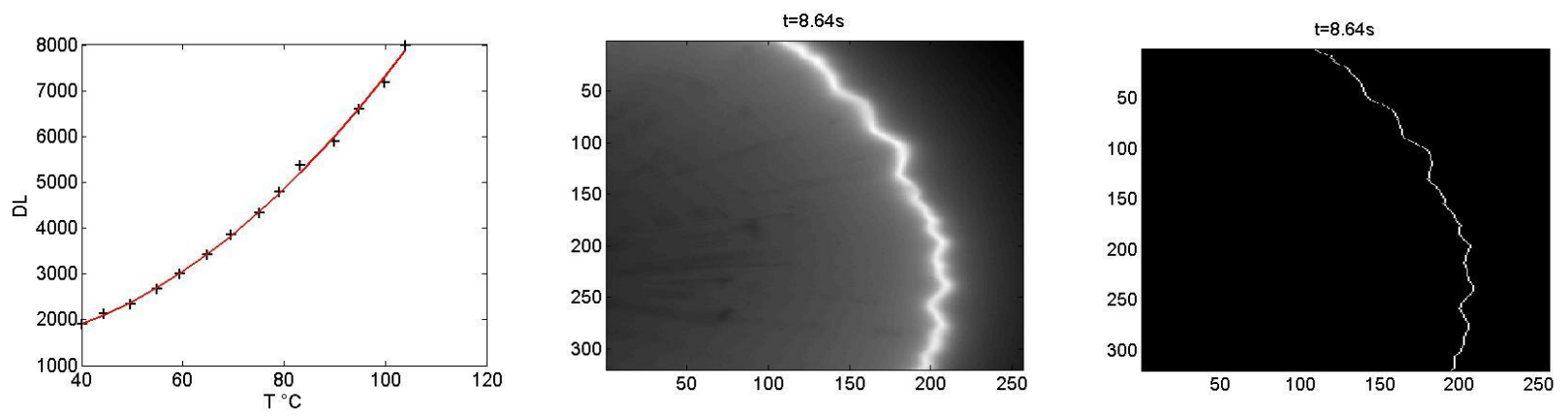

Fig. 4. Calibration curve used to convert $\mathrm{DL}$ into Celsius degrees (left side), temperature map at $\mathrm{t}=8.64 \mathrm{~s}$ (center), and solid-liquid interface position at $\mathrm{t}=8.64 \mathrm{~s}$ (right).

The graphical representation of $s(x, y, t)$ at the different sampling times (hereafter: map of the crystallization fronts) is then used to determine the trajectories and the velocity of the interface's points, as well as the temperature profiles along these trajectories. In practice, only a limited number of points is considered. For instance, in dendritic structures, the trajectory and the velocity of the tips of dendrites are typically analyzed. Figure 5 (left-top side) represents the map of the crystallization fronts corresponding to the experiment in Fig. 3. The trajectories of the tip of three dendrites are depicted in red, blue and green color. As expected for unconstrained crystal growth, these trajectories are straight lines that cross at point where the crystallization has been started. Knowing the equation of these lines, the temperature profiles along the trajectories can be easily retrieved from the temperature maps. Figure 5 (right-top side, bottom side) shows the temperature profile along the trajectories at different sampling times. For all times, the temperature profile shows a maximum at the solid-liquid interface (tip position) and progressively decreases with the distance to the interface, both in the crystal and in the liquid phase. Indeed, if the rate of production of latent heat due to the crystal growth is faster than the rate of removal by the heating/cooling device, the temperature at the interface will raise up and becomes higher than the bulk temperature, thus creating thermal gradients in the liquid and in the solid that contribute to the heat removal. If the crystal growth rate is constant, a pseudo steady-state regime is reached and the thermal profile along the trajectory of any point of the interface verify: $T(z, t)=T\left(z+v\left(t-t_{0}\right), t_{o}\right)$, where $z$ is the coordinate on the trajectory, $v$ is the point velocity and $t_{0}$ is an arbitrary chosen initial time.
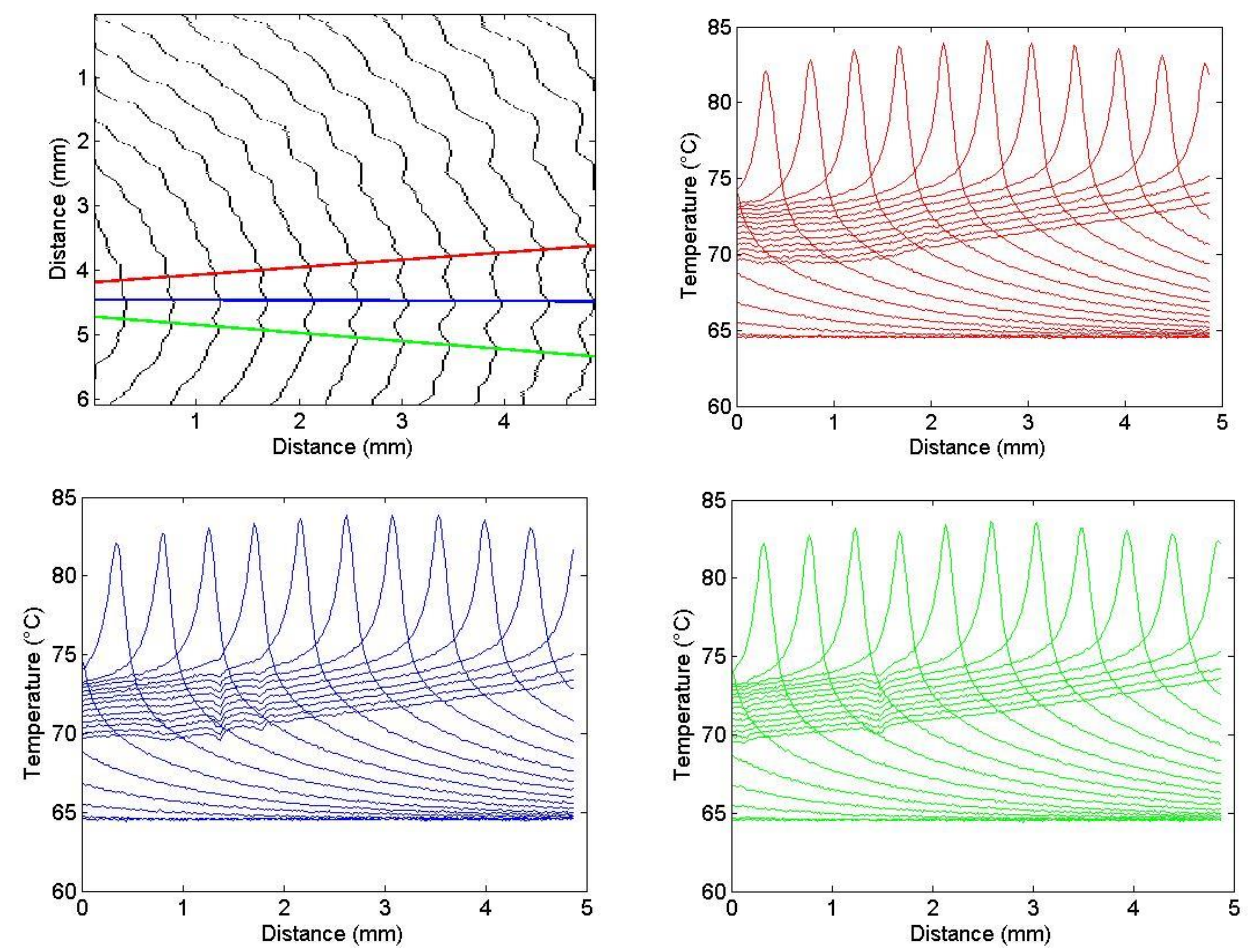

Fig. 5. Straight lines describing the trajectory of the tip of three dendrites (left-top side) and temperature profile along the trajectories at different sampling times (right-top, left-bottom, right-bottom).

To analyze the crystal growth kinetic, the distance traveled by the chosen points along their trajectories is calculated as a function of time, $d(t)$ (Fig.6). The velocity of the points is hence the time derivative of this function. If the crystal growth is controlled by diffusion, $d(t)$ is proportional to the square root of time. On the contrary, $d(t)$ is a linear function of time when 
the rate-controlling process is either the reaction at the crystal-melt interface or the flow of latent heat away from the interface. In these latter cases, the crystal growth velocity is hence constant and it is given by the slope of $d(t)$.

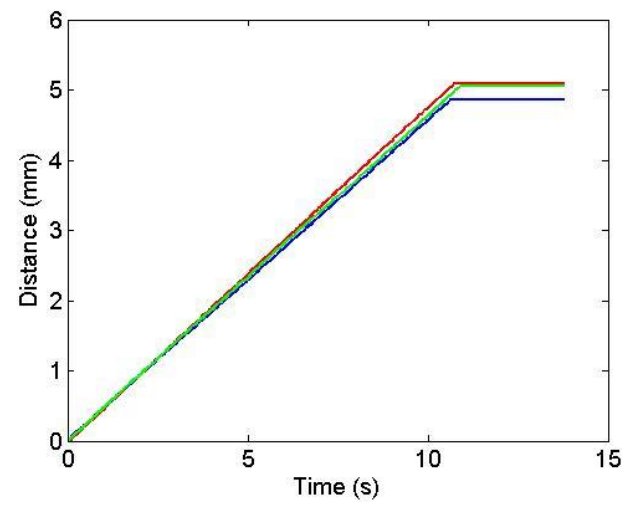

Fig. 6. Distance traveled by a point along each studied straight line; over time.

\section{Experimental analysis of erythritol crystal growth}

\subsection{Erythritol and experiments carried out}

Erythritol $\left(\mathrm{C}_{4} \mathrm{H}_{10} \mathrm{O}_{4}\right)$ is a sugar alcohol which is usually used as sugar replacer [8] and more recently as phase change material for thermal energy storage purposes [9]. Major thermodynamic and physical properties related with melting and solidification of erythritol have been measured in SAM.SSA project [10] and are summarized in Table 1.

Table 1. Properties of erythritol

Melting point: $T_{m}=118^{\circ} \mathrm{C} ;$ Latent heat: $\Delta H_{l s}=340 \mathrm{~J} / \mathrm{g}$

Density (liquid, $70^{\circ} \mathrm{C}<T<130^{\circ} \mathrm{C}$ ): $\rho=a T+b$ (g/cc), with $a=-0.001, b=1.48$

Density (solid, $30^{\circ} \mathrm{C}<T<110^{\circ} \mathrm{C}$ ): $\rho=a T+b\left(\mathrm{~g} / \mathrm{cc}\right.$ ), with $a=-8 \times 10^{-4}, b=1.5135$

Specific heat (liquid, $\left.40^{\circ} \mathrm{C}<T<130^{\circ} \mathrm{C}\right): c_{p}=a T^{2}+b T+c(\mathrm{~J} / \mathrm{g} / \mathrm{K})$, with $a=-1.741 \times 10^{-5}, b=5.88 \times 10^{-3}, c=2.696$

Specific heat (solid, $20^{\circ} C<T<110^{\circ} C$ ): $c_{p}=a T^{2}+b T+c(\mathrm{~J} / \mathrm{g} / \mathrm{K})$, with $a=8.509 \times 10^{11}, b=4.128 \times 10^{-3}, c=1.303$

Viscosity $\left(90^{\circ} C<T<130^{\circ} C\right): \eta=a \exp (b / T)$ (Pa.s), with $a=1.013 \times 10^{-3}, b=408.1$

The crystal growth of erythritol at different undercooling degrees has been investigated by the method described in sections 3 and 4 . The total number of experiments carried out is 14 , corresponding to different values of $\mathrm{T}_{\mathrm{o}}\left(41^{\circ} \mathrm{C}\right.$ to $106^{\circ} \mathrm{C}$ every $5^{\circ} \mathrm{C}$ ). The highest and lowest undercooling that have been investigated are $80^{\circ} \mathrm{C}$ and $17^{\circ} \mathrm{C}$ respectively. The samples are $300 \mu \mathrm{m}$ of thickness. Thicker samples $(650 \mu \mathrm{m})$ have also been tested, but no significant differences regarding growth rates have been observed. Figure 7 shows several snapshots of the infrared images recorded during the experiments carried out at $\mathrm{T}_{0}=41,51,61,71,81$ and $101^{\circ} \mathrm{C}$.
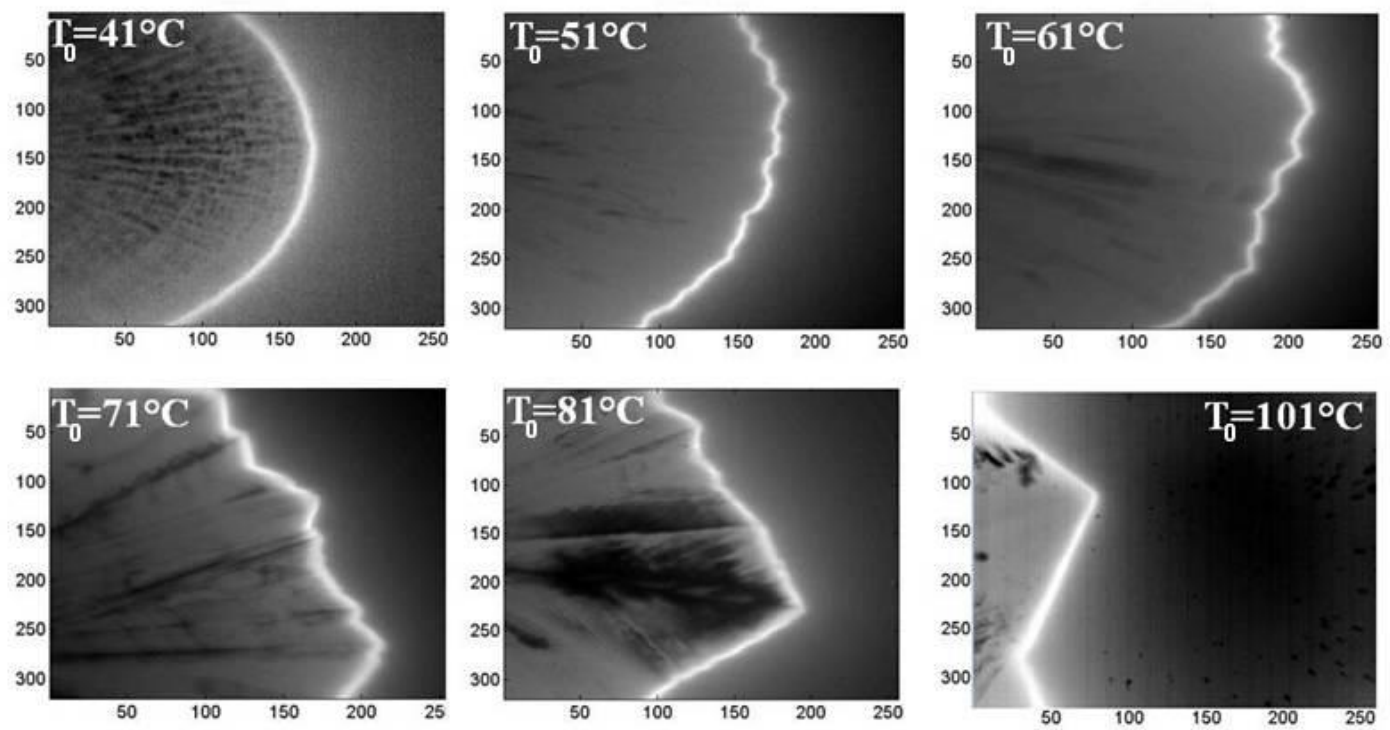

Fig.7. Snapshots of the infrared images recorded at different working temperatures 


\subsection{Crystal growth velocity}

Crystal growth velocities have been determined by the procedure described in section 4.2. Figure 8 (left side) shows the map of crystallization fronts for the experiments carried out at $T_{0}=41^{\circ} \mathrm{C}$ and $T_{0}=91^{\circ} \mathrm{C}$, as well as the trajectory of one of the points of the interface (red line). The temperature profile along this line is depicted on the right side of Fig. 8 for different sampling times (one curve by sampling time). Figure 5 corresponds to the experiment carried out at $\mathrm{T}_{0}=66^{\circ} \mathrm{C}$. Regarding trajectories and temperature profiles, the remarks in section 4.2 apply to all the tests carried out. The distance travelled $d(t)$ by the chosen points along its own trajectories increases linearly with time. The crystal growth velocity is constant and it is hence calculated as the slope of $d(t)$.

The crystal growth velocity calculated at different working temperatures is presented in Figure 9. Blue points represent the growth velocity against the undercooling at the interface $\left(T_{m}-T\right)$ (left side) and against the interface temperature $T$ (right side). It can be seen that the crystal growth velocity initially increases with the undercooling, reaches a maximum, and then slows down as the temperature is further decreased. This behaviour is in agreement with the theory of crystal growth for diffusion-limited kinetics (see section 2). At the melting point, melting and solidification are in equilibrium and the crystal growth velocity is hence zero. As the temperature is lowered, the driving force of crystallization grows and the erythritol crystallization occurs faster, until it becomes limited by the dynamics of molecular rearrangement. Diffusion is a thermally activated process and, therefore, the lower the temperature the lower the rate of diffusion. At sufficiently low temperature liquid mobility is effectively suppressed (e.g. below the glass transition temperature, $\mathrm{T}_{\mathrm{g}}$ ) so that the molecules are unable to undertake crystal formation. The crystal growth becomes hence zero.

In the same figures, red points correspond to the growth velocity represented against the undercooling in the bulk $\left(T_{m}-T_{0}\right)$ (left side) and the far-field temperature $T_{0}$. As expected, red points are shifted to lower temperatures compared to blue ones. This comparison shows that the measurement of temperature at the interface is essential when discussing the temperature dependence of experimentally determined growth rates.
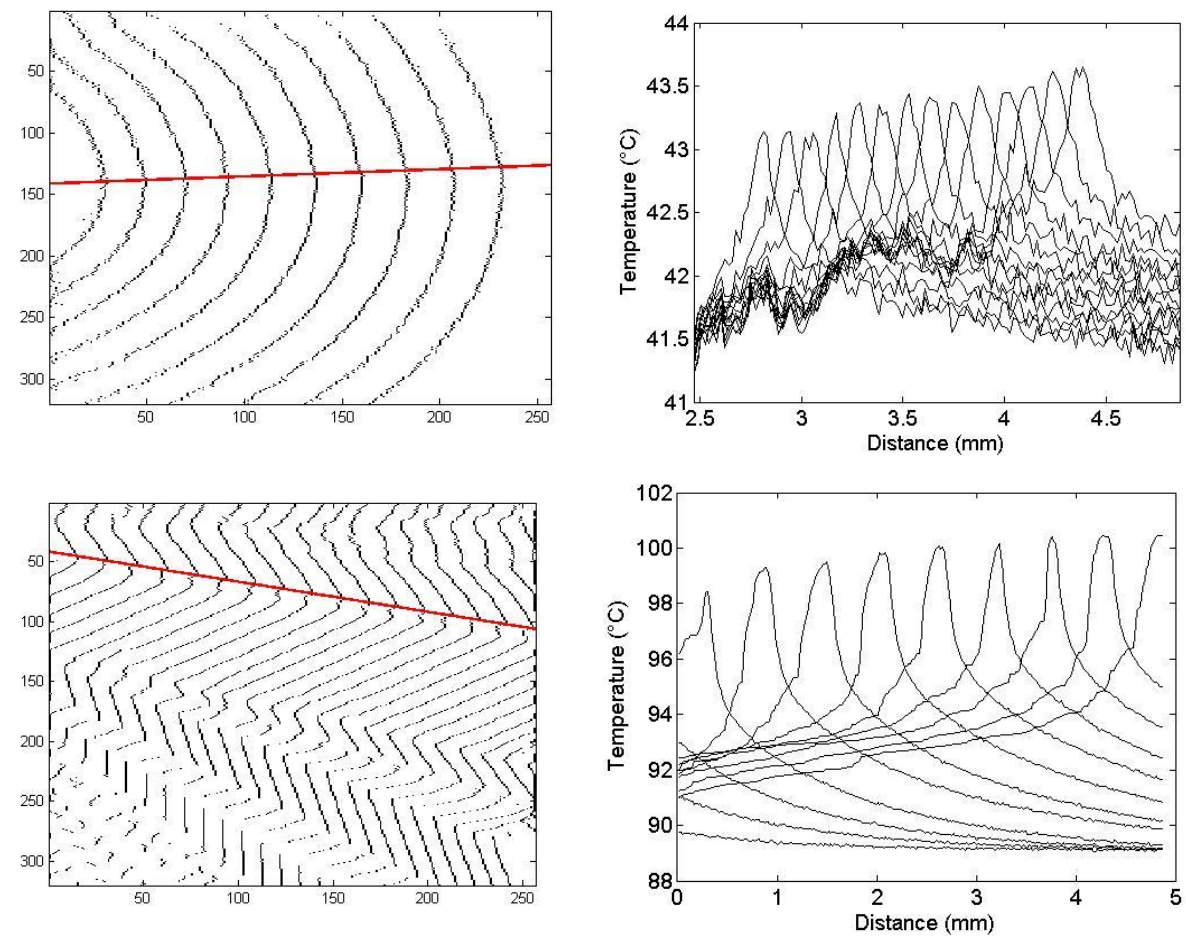

Fig. 8.Straight lines connecting the positions of dendrite tipsa) at $41^{\circ} \mathrm{C}, \mathrm{c}$ ) at $91^{\circ} \mathrm{C}$;evolution of the temperature along the red line at different time steps b) at $41^{\circ} \mathrm{C}$ d)at $\mathrm{T}=91^{\circ} \mathrm{C}$. 

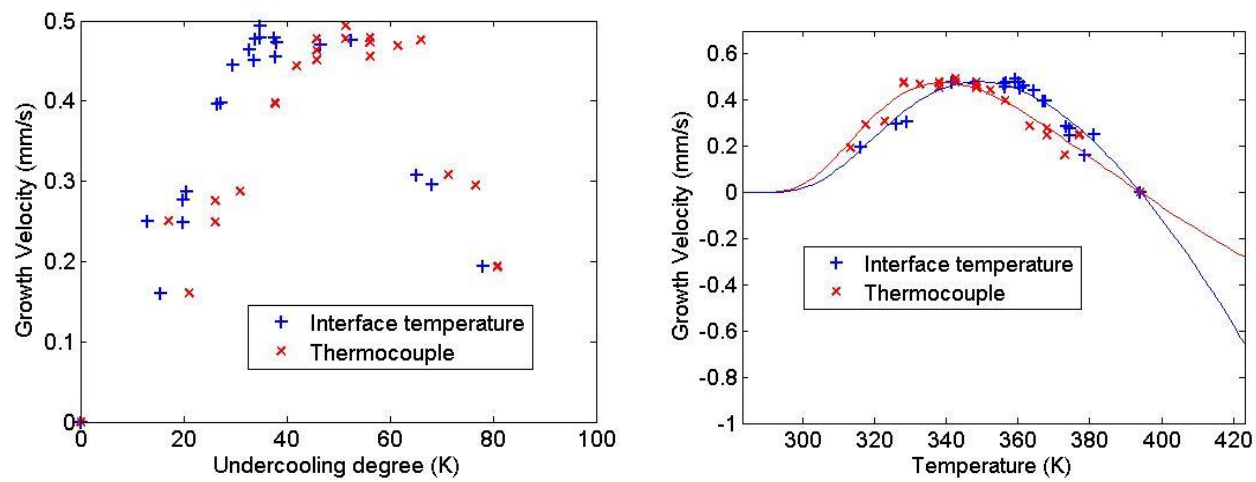

Fig. 9. Crystal growth velocity vs. undercooling (left side) and crystal growth velocity vs. temperature (right side). Blue points represent the growth velocity against the undercooling $T_{m}-T$ or the temperature $T$ at the crystal-melt interface, whereas bulk undercooling $T_{m}-T_{o}$ and bulk temperature $T_{o}$ are used for the red points.

\subsection{Thermodynamic analysis}

As already mentioned, the experiments carried out show that the erythritol crystal growth is interface-controlled with diffusion-limited kinetics. The modern form of the Wilson-Frenkel model for crystal growth hence applies and it is used here to investigate the temperature dependence of the fraction $f$ of interface sites that are active growth sites. Introducing equations (3) and (5) into Eq. (4) yields

$$
v=\frac{2 k_{B} T}{\pi \eta(T) l^{2}} f \exp \left(-\frac{\Delta S_{a}}{R}\right)\left[1-\exp \left(-\frac{\Delta H_{l s} \Delta T}{R T_{m} T}\right)\right]
$$

We can hence write

$$
k f=\frac{v \eta(T)}{\left(2 k_{B} T / \pi\right)\left[1-\exp \left(-\frac{\Delta H_{l s} \Delta T}{R T_{m} T}\right)\right]} \quad \text { with } k=\frac{1}{l^{2}} \exp \left(-\frac{\Delta S_{a}}{R}\right)
$$

By assuming that the entropy difference, $\Delta S_{a}$, and the diffusion jump distance, $I$, are not sensitive functions of the temperature, the pre-factor $k$ can be considered as a constant and Eq. 10 can be used to analyze the temperature dependence of $f$. This equation can also be used to distinguish which mechanism controls the interface reaction, because $f$ has a different temperature dependence for each mechanism. The mechanism of growth may be defined as the manner in which atoms or molecular groups attach to the growing crystal surface. Two broad categories of mechanisms can be distinguished: lateral and continuous. The continuous mechanism operates when molecules can attach to the crystal surface at essentially any site (rough surfaces), allowing the interface to advance uniformly. For continuous growth $f$ is generally assumed to be independent of the temperature and large. Lateral growth occur when the surface of a crystal is essentially atomically flat (smooth surfaces). Two idealized types of lateral mechanisms can be distinguished: surface-nucleation and screw dislocation. In the surface-nucleation mechanism it is assumed that molecules can attach only at the edges of one-molecule-thick layer on the crystal surface. Each layer is initiated by one or more one-molecule-thick nucleii, the formation of which obeys the classical laws of nucleation kinetics. In such case, the density of active growth sites $f$ depends on the density of growth sites provided by the nucleation process. However, defects in the crystal structure can help to form new layers, so that a nucleation process is not necessary for growth. For instance, a screw dislocation that ended at a surface provides a continuous step on a surface for growth. The fraction $f$ of active sites for growth is now proportional to the undercooling [6].

Using the measured values for the crystal growth velocity $v$ (data in Fig. 9), the values of melting point $T_{\mathrm{m}}$ and latent heat of fusion $\Delta H_{I s}$ of erythritol and the viscosity function $\eta(T)$ given in Table 1, the temperature dependence of $f$ has been calculated by using Eq. 10. The results achieved are depicted in Figure 10. 

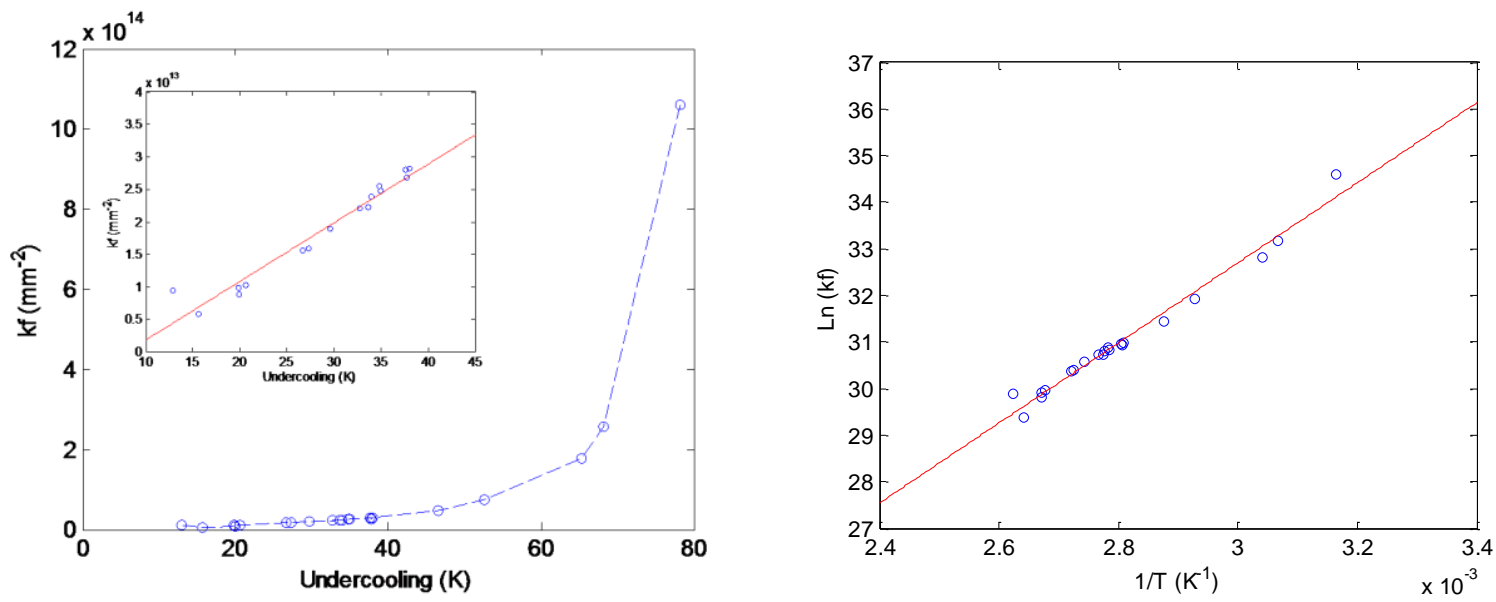

Fig. 10. Temperature dependence of the density of active growth sites.

As shown in Fig. 10 (left side, inset), at small uncercoolings $\left(T_{m}-T<45^{\circ} \mathrm{C}\right)$ the fraction $f$ of active sites for growth increases linearly with the undercooling, in accordance with the theory of growth on screw dislocations. Then the density of active growth sites increases dramatically. At small undercoolings the defect density determines the density of active growth sites, and at large undercooling another mechanism takes over. This second mechanism could be either surface nucleation or a continuous mechanism due to increased roughness of the edge of the steps or both. As it can be seen in Fig. 7, the morphology of the crystal-melt interface is also changing with undercooling. At small undercoolings ( $T_{m}-T<$ $40^{\circ} \mathrm{C}$ ), faceted interfaces with large facets are observed. With progressively larger undercooling, the size of the facets decreases. At high undercooling $\left(T_{m}-T>60^{\circ} \mathrm{C}\right)$, the morphology of the interface is spherulitic. Both figures, Fig. 7 and Fig. 10 , indicate that the surface of the erythritol crystals is essentially atomically flat and that the mechanism of growth is dominated by defects at small undercoolings $\left(T_{m}-T<45^{\circ} \mathrm{C}\right)$. At intermediate undercoolings, the wavelengths of the instabilities at the interface decrease. The interface still is faceted but the facets are smaller and the roughness of the interface is probably higher. At high undercooling $\left(T_{m}-T>60^{\circ} \mathrm{C}\right)$, the morphology of the interface becomes spherulitic and its roughness is probably also increased.

In Fig. 10 (right side) is represented the logarithm of $k f$ against to the inverse of temperature. It can be seen that there is a quite good linear relationship between $k f$ and $1 / T$. Therefore, the following model has been adjusted to the data (red line in Fig. 10):

$$
k f=C_{1} \exp \left(\frac{C_{2}}{T}\right)
$$

with $\mathrm{C}_{1}=1017\left(\mathrm{~mm}^{-2}\right)$ and $\mathrm{C}_{2}=8591.8\left(\mathrm{~K}^{-1}\right)$. The crystal growth velocity is hence approached by

$$
v=C_{1} \exp \left(\frac{C_{2}}{T}\right) \frac{2 k_{B} T}{\pi \eta(T)}\left[1-\exp \left(-\frac{\Delta H_{l s} \Delta T}{R T_{m} T}\right)\right]
$$

The blue line in Fig. 9 (right side) represents the crystal growth velocity calculated by Eq.(12). The agreement with measurements is quite good. Once again we notice the importance of a detailed analysis of the interface temperature when discussing the temperature dependence of experimentally determined growth rates. Indeed, the red line in Fig. 9 (right side) corresponds to Eq. (12) in which $C_{1}, C_{2}$ and $v$ are obtained using the values of the bulk temperature (far field temperature $T_{0}$ ) instead of the interface temperature $T$. Although the agreement between measurements and predictions still is good, it can be observed that at temperature above the melting point the growth rate does not decrease rapidly to minus infinity as it must be.

\section{Conclusion}

A new experimental approach based on infrared thermography has been proposed for studying crystal growth kinetics in undercooled melts. The crystallization of a thin sample of an undercooled melt at constant bulk temperature is induced by a small crystal seed and the growth of the solid phase is observed using infrared camera. The recorded thermal images allow determining the position of crystallization front at any time, the velocity of advancement of the front at any point and any time, and the temperature at the points of the interface at any time. Contrary to experimental techniques based on optical microscopy or video cameras, infrared thermography provides detailed analysis of the interface temperature which is essential when discussing the temperature dependence of experimentally determined growth rates. The appropriateness of infrared thermography for crystal growth kinetics analysis has been illustrated through the experimental analysis of erythritol crystallization. Although experimental techniques based on optical microscopy or video 
cameras provide a better view of the crystal morphology than infrared thermography, it has been proven that the latter one is better for analyzing the temperature dependence of crystal growth rates. Moreover, infrared thermography can also be used for studying specimens which are not transparent to visible light.

\section{Acknoledgment}

The authors acknowledge the financial support of the European Commission for subsidizing SAM.SSA (Sugar Alcohols based Materials for Seasonal Storage Applications) Project within the 7th framework program for research.

\section{REFERENCES}

[1] Asta M. et al., "Solidification microstructures and solid-state parallels: Recent developments, future directions", Acta Materialia, 57, pp. 941-971, 2009.

[2] Verma S., Shlichta P.J., "Imaging techniques for mapping solution parameters, growth rate, and surface features during the growth of crystals from solution", Progress in Crystal Growth and Characterization of Materials, 54, pp.1$120,2008$.

[3] Wilcox W.R., Shlichta P.J., "A survey of movies on crystal growth", Journal of Crystal Growth , 15, pp.61-72, 1972.

[4] Carignano M.A., Shepson P.B., Szleifer I., "Molecular dynamics simulations of ice growth from supercooled water", Molecular Physics, 103 (21-23), pp. 2957-2967, 2005.

[5] Rozmanov D. and Kusalik P.G., "Temperature dependence of crystal growth of hexagonal ice", Phys. Chem. Chem. Phys, 13, pp. 15501-15511, 2011

[6] Jackson K.A., "Kinetics processes. Crystal Growth, Diffusion, and Phase Transitions in Materials", Wiley-VCH Verlag GmbH \& Co. KGaA, Weinheim, 2004.

[7] Herlach D.M., Galenko P., Holland-Moritz D., "Metastable Solids from Undercooled Melts", Pergamon Materials Series, Amsterdam, 2007.

[8] Livesey G., "Health potential of polyols as sugar replacers, with emphasis on low glycaemic properties", Nutrition Research Reviews, 16, pp. 163-191, 2003.

[9] Lee S-Y., Park M., Park S-J., "Thermal characterization of erythritol/expanded composites for high thermal storage capacity", Carbon, DOI: http://dx.doi.org/10.1016/j.carbon.2013.09.053

[10] Godin A., Palomo del Barrio E., Duquesne M., Daranlot J., Tadmouri R., Pavageau B., van Wissen R.M.J, GaastraNedea S., Zhang H., "Summary of measured physical properties", 4th Progress Meeting, FP7 project-SAM.SSAWork Package 3, Bucharest, April 2014. 